\section{Rapid progressive seeding of a community acquired pathogen in an immune-competent host: end organ damage from head to bone}

\author{
Daisy Torres-Miranda, Farah Al-Saffar, \\ Saif Ibrahim, Stephanie Diaz-Font \\ Department of Internal Medicine, UF \\ Health Jacksonville, FL, USA
}

\begin{abstract}
Methicillin-sensitive Staphylococcus aureus (MSSA) meningitis is a rare disease when not related to neurosurgery: there are only few reported cases in the literature to date. We describe a case that highlights not only meningeal but also diffuse and rapidly progressive systemic involvement with multi-organ failure. A 64-year-old male presented to our hospital with a chief complaint of acute worsening of his usual chronic lower back pain, progressive weakness in lower extremities and subjective fevers at home. Hospital course demonstrated MSSA bacteremia, of questionable source, that resulted in endocarditis affecting right and left heart in a patient with no history of intravenous drug use. The case was complicated by septic emboli to systemic circulation involving the kidneys, vertebral spine, lungs and brain with consequent meningitis and stroke, even when treated empirically with vancomycin and then switched to nafcillin as indicated. Even though MSSA infections are well known, there are very few case reports describing such an acute-simultaneous-manifestation of multiend-organ failure, including meningitis and stroke. Our case, also presented with an uncommon manifestation of persistent infection dissemination despite adequate antibiotic treatment.
\end{abstract}

\section{Introduction}

Methicillin-sensitive Staphylococcus aureus is a gram-positive cocci in clusters. It is carried in anterior nares by $20-30 \%$ of population. Higher carriage rates are seen in diabetics, intravenous drug users (IVDU), HIV and dialysis patients. ${ }^{1,2}$ All patients with $S$. aureus bacteremia should undergo transthoracic echocardiography (TTE), since $S$. aureus bacteremia is associated with heart valve involvement in $25 \%$ of the cases. ${ }^{3}$ Nevertheless, TEE has been shown to be superior to TTE for the diagnosis of infective endocarditis (IE), identifying small vegetations and abscesses ${ }^{3,4}$ All infective foci must be identified and removed as soon as possible; however, foci are not always obvious and long-term antimicrobial therapy might be necessary. Mortality associated with $S$. aureus bacteremia is $20-40 \% .^{5}$ Nafcillin is a wellestablished agent for serious systemic non methicillin-resistant $S$. aureus (MRSA) infections and has been reported as superior over vancomycin. ${ }^{6-9}$ We present a very interesting case of a rapidly progressive methicillin-sensitive Staphylococcus aureus infection, for which empirical treatment with vancomycin and initial treatment with nafcillin did not stop further dissemination despite adequate MIC, taking longer than usual to respond to adequate treatment.

\section{Case Report}

A 64-year-old male patient with a past medical history of hypertension, hiatal hernia and osteoarthritis presented to the emergency department with a chief complaint of acute worsening of his chronic lower back pain for two weeks and progressive weakness in lower extremities. He used to ambulate with a cane and later used a walker for several days, but recently he felt non-ambulant. He also described subjective fevers at home. Physical examination was significant for temperature of $100.1^{\circ} \mathrm{F}$. Blood pressure, respiratory and heart rate were within normal limits. He appeared in no distress, cachectic and disheveled with poor dentition. Cardiac examination showed a regular rate and rhythm with normal S1 and S2 and no murmurs. Lungs were clear to auscultation with bilateral air entry. He had crepitus in both knees and a limping gait. Kernig's sign and Brudzinski's sign were obscured by back pain. On further review of systems he reported having chronic bilateral knee pain related with osteoarthritis and a congenital deformation of his right knee. He was a smoker of 40 pack-years, occasional user of alcohol and marijuana, but denied ever using intravenous drugs and toxicology was positive only for oxycodone, which he used for chronic lumbar pain for several years.

Laboratory exams displayed a leukocytosis of 25,500 with $89 \%$ neutrophils, no bands, sedimentation rate of 44 , lactic acid 1.6, anion gap 18. Thoracic and lumbar spine computed tomography (CT) scan showed multilevel central canal and bilateral neural foraminal compromise, but did not show evidence of abscess. Partially visualized lungs showed a cavitary lesion in the superior segment of the left lower lobe, measuring $1.4 \mathrm{~cm}$ with circumferential thick wall, suggestive of septic emboli versus tuberculosis, and left inferior renal pole abnor-
Correspondence: Daisy Torres-Miranda Department of Internal Medicine, UF Health Jacksonville, $655 \mathrm{~N}$ 8th Street, Jacksonville, FL 32209 , USA.

Tel.: + 1.787.402.9550.

E-mail: daisy.torres-miranda@jax.ufl.edu

Key words: Bacteremia; septic emboli; Methicillin sensitive Staphylococcus aureus.

Contributions: the authors contributed equally.

Conflict of interest: the authors declare no potential conflict of interest.

Conference presentation: this case was presented in the American Thoracic Society Conference; May 2015.

Received for publication: 4 February 2015.

Revision received: 24 March 2015.

Accepted for publication: 26 March 2015.

This work is licensed under a Creative Commons Attribution NonCommercial 3.0 License (CC BYNC 3.0).

(C) Copyright D. Torres-Miranda et al., 2015

Licensee PAGEPress, Italy

Infectious Disease Reports 2015; 7:5849

doi:10.4081/idr.2015.5849

malities suggestive of multiple infarctions. Blood cultures grew $S$. aureus.

The patient was started empirically on vancomycin $15 \mathrm{mg} / \mathrm{kg} \mathrm{IV} \mathrm{q} 12 \mathrm{~h}$, while waiting for sensitivities. The TTE performed after bacteremia diagnosis showed an ejection fraction of $65 \%$ with normal valves and no vegetations. On day two of hospitalization the clinical picture worsened as the patient suddenly developed an altered mental status and nuchal rigidity. Lumbar puncture confirmed meningitis with a cerebrospinal fluid leukocytosis of 1157 (neutrophil 95\%) and culture positive for $S$. aureus. Testing for HIV, Herpes Simplex Virus (HSV) and Tuberculin skin test (PPD) were all negative. Spine magnetic resonance imaging (MRI) showed osteomyelitis at T12-L1 and previously seen (in CT scan) renal infarcts. The patient continued to be febrile despite pathogen susceptible to vancomycin with MIC $<2 \mathrm{mg} / \mathrm{mL}$, trough previous to $4^{\text {th }}$ dose 11, repeated trough 18.4 , repeat blood cultures at 48 and 96 hours remained negative. Six days later he had clinical deterioration with tachypnea, hypoxia, new systolic 2/6 murmur, louder over cardiac apex area, and bilateral respiratory crackles. He also developed new right hemiparesis with upgoing babinski reflex of the right side. At this point, the patient was switched to nafcillin $2 \mathrm{~g}$ IV q $4 \mathrm{~h}$ when blood culture results confirmed methicillin susceptibility on day 3 of admission. Head MRI showed multiple infarcts in a non- 
vascular pattern secondary to septic embolisms (Figure 1). The TEE showed severe mitral and tricuspid regurgitations, with 1.5 $\mathrm{cm}$ mobile vegetation on the posterior leaflet of the mitral valve. The patient was transferred to the intensive care unit due to the complicated picture of MSSA bacteremia, IE, osteomyelitis, meningitis, ischemic stroke, renal and pulmonary infarcts secondary to septic emboli. Nafcillin was continued with appropriate resolution of leukocytosis and fever. Patient's mental status slowly improved and returned to baseline. There were indications for emergent mitral valve replacement, however given his recent finding of embolic stroke; this was not feasible due to high mortality risk. Follow up TTE showed worsening mitral and tricuspid valve involvement, therefore mitral and tricuspid valve replacements were performed, four weeks from IE diagnosis. He completed 8 weeks of nafcillin (given his vertebral involvement and unknown source). After 2 months of hospitalization, patient was dis-

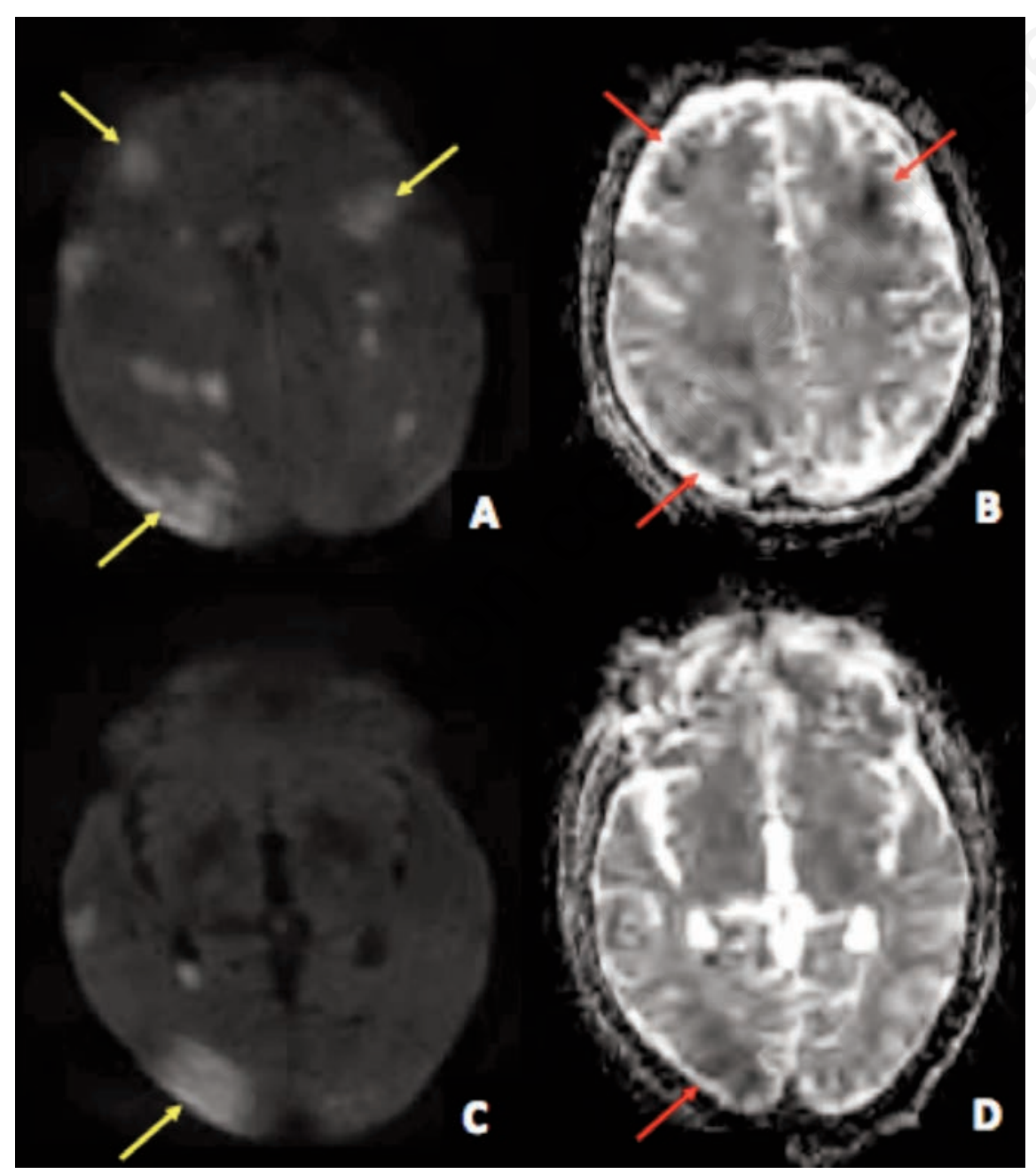

Figure 1. A) Axial diffusion weighted image shows multifocal intra-parenchymal hyperintensities in a non-vascular pattern with $(B)$ corresponding hypo-intense apparent diffusion coefficient findings. C) and D) right occipital restricted diffusion. Findings are compatible with acute multifocal ischemia secondary to septic embolisms. charged home with a dual-chamber pacemaker due to persistent 3rd degree atrio-ventricular block, post surgery.

\section{Discussion}

Staphylococcus aureus is one of the leading agents of infection among adult patients. When $S$. aureus invades deep structures it often metastasize hematogenously to other areas and organs with significant morbidity. ${ }^{10}$ Infection caused by community acquired MSSA strains are characterized by severe clinical course with increased incidence of endocarditis and organ failure. A bacterial origin is not always obvious. Two important questions should be asked to the patient with the intention to identify focal source and consider additional necessary diagnostic test. Those are: use of intravenous drugs and presence of painful joints. IVDU related infection can man- ifest as an initially insidious presentation that later complicates to be an aggressive metastatic disease. ${ }^{11}$

We presented a unique case since our patient was not an IVDU nor an initial focus of infection was identified. Additionally, the progression from negative to positive echocardiogram findings of valvular vegetations highlights the high virulence of this communityacquired pathogen, since severe valvular regurgitation or insufficiency should be equally observed on both types of echocardiograms. Altered mental status in such patients should alert the physicians to the possibility of brain emboli causing multifocal ischemia. A MRI is crucial to confirm the diagnosis with the visualization of a non-vascular pattern..$^{12,13}$ Meningitis due to $S$. aureus is uncommon, when not related to neurosurgery. MSSA meningitis is a serious infection, which can occurs in patients without risk factors or immunosuppression. Like meningitis, stroke secondary CA-MSSA is rarely seen, regardless of the severity of infection. High level of clinical suspicion is needed in such patients, as back pain could be the only reliable predictor of an added spine infection. ${ }^{14,15}$

When treating for gram-positive cocci bacteremia, empirical antibiotic therapy should provide coverage against staphylococci, usually with vancomycin to cover MRSA. ${ }^{15}$ Nafcillin/oxacillin remains the antibiotic of choice for treating infections caused by MSSA once culture and sensitivity results confirm it, because nafcillin is superior to vancomycin in preventing persistent or relapsing MSSA bacteremia. ${ }^{10}$ In the case of endocarditis, studies have demonstrated that vancomycin (versus nafcillin) is significantly associated with relapse given its slow bactericidal activity. ${ }^{5,6}$ For our case, the reasons why empirical treatment with vancomycin and initial treatment with nafcillin, did not stop further dissemination remained unknown. Nevertheless, continued treatment with nafcillin eventually did resolve the infection. We have to consider that, even though the MIC remains the only satisfactory in vitro measurement of the intrinsic activity of antimicrobials, the test has always been open for criticism since it is performed with the use of artificial media and fixed concentrations, under conditions that may be very different from those in the actual site of infection. ${ }^{16}$ There is evidence that support the concept of the principal determinant of efficacy of beta lactams to be the time for which the drug levels exceed the MIC at the site of infection, not just and MIC $<2 \mathrm{mg} / \mathrm{mL}$ by itself. Also, bacterial strains have been detected to have a MBC (minimum bactericidal concentration) many times higher than the MIC, (known as phenotypic tolerance), isolated in vitro grampositive bacteria causing slower clinical response. ${ }^{16-18}$ Another speculative answer 
would be the inoculum effect. The inoculum effect may be clinically relevant since the number of bacteria at the site of infection may much higher that the traditionally used for susceptibility testing. ${ }^{16,19}$ Unfortunately, molecular genotyping or DNA techniques were not performed.

In our case, specific nafcillin concentrations were not targeted. Although the benefit of such concentration is questionable since our patient's infection was multifocal. Our laboratory was not able to analyze serum nafcillin concentrations; hence, samples would have to send to a referral laboratory.

\section{Conclusions}

A community acquired pathogen, even when part of the normal flora could be virulent enough to cause end organ damage from head to toe. The use of head MRI as a pre evaluation tool for IE-related urgent valve surgery is imperative, to investigate whether such preoperative findings affect postoperative outcomes. The presence of characteristic cranial MRI lesions may prompt early diagnosis of infective source and lead to the adequate management. Beyond the MIC, the time the drug level exceeds the MIC, the phenotypic tolerance and the inoculum effects may be reasons why empirical treatment with vancomycin and initial treatment with nafcillin did not stop further dissemination, but, eventually, clearing the infection.

\section{References}

1. von Eiff C, Becker K, Machka K, et al. Nasal carriage as a source of Staphylococcus aureus bacteremia. Study Group. N Engl J Med 2001;344:11-6.

2. Simor AE. Staphylococcal decolonisation: an effective strategy for prevention of infection? Lancet Infect Dis 2011;11:95262.

3. Baddour LM, Wilson WR, Bayer AS, et al. Infective endocarditis: diagnosis, antimicrobial therapy, and management of complications: a statement for healthcare professionals from the Committee on Rheumatic Fever, Endocarditis, and Kawasaki Disease, Council on Cardiovascular Disease in the Young, and the Councils on Clinical Cardiology, Stroke, and Cardiovascular Surgery and Anesthesia, American Heart Association: endorsed by the Infectious Diseases Society of America. Circulation 2005;111:394-434.

4. Fowler VG Jr, Li J, Boley J, et al., Role of echocardiography in evaluation of patients with Staphylococcus aureus bacteremia: experience in 103 patients. J Am Coll Cardiol 1997;30:1072-8.

5. Thwaites GE, Edgeworth JD, GkraniaKlotsas E, et al. Clinical management of Staphylococcus aureus bacteraemia. Lancet Infect Dis 2011;208-22.

6. Valour F, Bouaziz A, Arsenty J, et al., Determinants of methicillin-susceptible Staphylococcus aureus native bone and joint infection treatment failure: a retrospective cohort study. BMC Infect Dis 2014;14:443.

7. Chang FY, Peacock JE, Musher DM, et al. Staphylococcus aureus bacteremia: recurrence and the impact of antibiotic treatment in a prospective multicenter study. Medicine (Baltimore) 2003;82:333-9.

8. Sung-Han K, Kye-Hyug K, Hong-Bin K, et al. Outcome of vancomycin treatment in patients with methicillin-susceptible Staphylococcus aureus bacteremia. Antimicrob Agents Chemother 2008;52: 192-7.

9. Leonard S. Synergy between vancomycin and nafcillin against Staphylococcus aureus in an in vitro pharmacokinetic/ pharmacodynamic. Plos One 2012;7: e42103.
10. Chang WN, Lu $\mathrm{CH}, \mathrm{Wu}$ JJ, et al. Staphylococcus aureus meningitis in adults: a clinical comparison of infections caused by methicillin-resistant and methicillin-sensitive strains. Infection 2001;29:245-50.

11. Ji Y, Kujtan L, Kershner D. Acute endocarditis in intravenous drug users: a case report and literature review. J Community Hosp Intern Med Perspect 2012;2.

12. Bakshi R, Wright PD, Kinkel PR, et al. Cranial magnetic resonance imaging findings in bacterial endocarditis: the neuroimaging spectrum of septic brain embolization demonstrated in twelve patients. J Neuroimaging 1999;9:78-84.

13. Novy E, Sonneville M, Mazighi IF, et al. Neurological complications of infective endocarditis: new breakthroughs in diagnosis and management. Med Mal Infect 2013;43:443-50.

14. Darouiche R. Spinal epidural abscess. N Engl J Med 2006;355:2012-20.

15. Ziu M, Dengler B, Cordell D, Bartanusz V. Diagnosis and management of primary pyogenic spinal infections in intravenous recreational drug users. Neurosurg Focus 2014;37:e3.

16. Turnidge J. The pharmacodynamics of beta-lactams. Clin Infect Dis 1998;27:1022.

17. Toumanen E, Durack D, Tomasz A, Antibiotic tolerance among clinical isolates of bacteria. Antimicrob Agents Chemother 1986;30:521-7.

18. Rahal J, Chan Y, Johnson G. Relationship of staphylococcal tolerance, teichoic acid antibody, and serum bactericidal activity to therapeutic outcome in Staphylococcus aureus bacteremia. Am J Med 1986;81:4352.

19. Gerber A, Greter U, Segessenmann C, Kozak $\mathrm{S}$. The impact of the pre-treatment interval on antimicrobial efficacy in a biological model. J Antimicrob Chemother 1993;31:29-39. 